\title{
A Microcircuit Model of the Frontal Eye Fields
}

\author{
Jakob Heinzle, Klaus Hepp, and Kevan A. C. Martin \\ Institute of Neuroinformatics, University and Swiss Federal Institute of Technology (ETH) Zürich, 8057 Zürich, Switzerland
}

\begin{abstract}
The cortical control of eye movements is highly sophisticated. Not only can eye movements be made to the most salient target in a visual scene, but they can also be controlled by top-down rules as is required for visual search or reading. The cortical area called frontal eye fields (FEF) has been shown to play a key role in the visual to oculomotor transformations in tasks requiring an eye movement pattern that is not completely reactive, but follows a previously learned rule. The layered, local cortical circuit, which provides the anatomical substrate for all cortical computation, has been studied extensively in primary sensory cortex. These studies led to the concept of a "canonical circuit" for neocortex (Douglas et al., 1989; Douglas and Martin, 1991), which proposes that all areas of neocortex share a common basic circuit. However, it has not ever been explored whether in principle the detailed canonical circuit derived from cat area 17 (Binzegger et al., 2004) could implement the quite different functions of prefrontal cortex. Here, we show that the canonical circuit can, with a few modifications, model the primate FEF. The spike-based network of integrate-and-fire neurons was tested in tasks that were used in electrophysiological experiments in behaving macaque monkeys. The dynamics of the model matched those of neurons observed in the FEF, and the behavioral results matched those observed in psychophysical experiments. The close relationship between the model and the cortical architecture allows a detailed comparison of the simulation results with physiological data and predicts details of the anatomical circuit of the FEF.
\end{abstract}

Key words: cortex; frontal eye field; laminar architecture; microcircuit; model; saccades

\section{Introduction}

The frontal eye field (FEF) of the monkey is a functionally well studied area. Electrical stimulation (Ferrier, 1874; Robinson and Fuchs, 1969; Bruce et al., 1985), recordings of neuronal activity (Bizzi, 1967; Bruce and Goldberg, 1985), and lesion studies (Dias et al., 1995; Dias and Segraves, 1999) demonstrate that the FEF is one of the key areas for processing saccadic eye movements. Visual responses and saccade vectors are topographically represented in the FEF (Robinson and Fuchs, 1969; Bruce and Goldberg, 1985). Neurons in the FEF show responses related to visual saliency and visual selection (Mohler et al., 1973; Bruce and Goldberg, 1985; Schall et al., 1995b), motor preparation (Segraves and Park, 1993), attention (Moore and Fallah, 2004; Schall, 2004; Thompson et al., 2005a), working memory (Goldberg and Bruce, 1990), and fixation (Hanes et al., 1998; Hasegawa et al., 2004).

Although much is known about its physiology and its connections with other cortical areas (Huerta et al., 1987; Schall et al., 1995a; Lynch and Tian, 2005) and subcortical structures (Huerta et al., 1986; Parthasarathy et al., 1992), the local cortical circuit of the FEF is not known. The major output of the FEF is to the superior colliculus (SC) (Leichnetz et al., 1981) and to premotor neurons in the reticular formation (Schnyder et al., 1985). Cyto-

\footnotetext{
Received March 5, 2007; revised June 7, 2007; accepted July 6, 2007.

This work was supported by Swiss National Science Foundation National Centres of Competence in Research Neural Plasticity and Repair and by European Union Daisy Project Grant FP6-2005-015803. We thank E. Salinas for sharing the code of his model (Salinas, 2003), parts of which were adapted for our simulations, and two anonymous reviewers for their helpful remarks.

Correspondence should be addressed to Jakob Heinzle at his present address: Bernstein Center for Computational Neuroscience, Philippstrasse 13/Haus 6, D-10115 Berlin, Germany. E-mail: jakob.heinzle@bccn-berlin.de. DOI:10.1523/JNEUROSCI.0974-07.2007

Copyright $\odot 2007$ Society for Neuroscience $\quad 0270-6474 / 07 / 279341-13 \$ 15.00 / 0$
}

architectonic comparisons of prefrontal granular cortex (Stanton et al., 1989; Petrides, 2005) and the connection patterns of single neurons in early visual (Kisvarday et al., 1989) (for review, see Douglas and Martin, 2004) and prefrontal (Kritzer and Goldman-Rakic, 1995) cortex suggest that the structure of the FEF might be similar to that of early visual areas.

Several models of the FEF have been proposed. An early study modeled the complete visual to oculomotor transformation including area FEF (Dominey and Arbib, 1992). More recently, three models specifically addressed the role of the FEF (Mitchell and Zipser, 2003; Brown et al., 2004; Hamker, 2005). All these models used rate coding rather than spiking neurons and took a rather simplified view of the local cortical circuitry.

Here we present a model of the local circuit of area FEF that follows the well described layered architecture of the neocortex and obeys the principles of a "canonical circuit" whose feature is its recurrent connections (Douglas et al., 1989; Douglas and Martin, 2004). We constructed a network of integrate-and-fire (IF) neurons based on a quantitative study of the connection matrix of cat area 17 (Binzegger et al., 2004) and tested whether it could replicate the electrophysiological and behavioral findings reported for FEF. The FEF model simulated several classical paradigms such as visual saccades and delayed memory saccades and also successfully performed a task that flexibly required either saccades or antisaccades. Hence, the canonical circuit model derived from cat primary visual cortex successfully captured the functionality of the primate FEF.

\section{Materials and Methods}

The local circuit model of the FEF presented here simulated the layered structure of neocortex. The model will first be explained by its functional 
architecture, and then the details of the IF neurons, synapses, and the pattern of connections will be described.

Separation of functions between layers. The control of saccadic eye movements requires several computational steps: selection of a target, allocation of attention to the location of the intended target, and the motor output that drives the eye movements. The selection of the next target could follow a particular rule, as in an antisaccade task or in reading. In addition, the oculomotor part of eye movement control interacts with cognitive processes that recognize visual features and influence how long attention is needed at a specific position. Here, we did not model such cognitive processes in detail but condensed them in a cortical module called REC (for recognition), which interacted with the FEF. The FEF model circuit received two external inputs. A retinotopic visual input, which represented the input from earlier visual areas, and a fixation input that was active when a fixation stimulus was present.

Figure 1 sketches the layers of the FEF network and explains their role in the visual-tooculomotor transformation. Only the feedforward connections within the network are shown. The arrows represent the general flow of information, but were not necessarily the strongest connections in the circuit. Layer 4 neurons received a dorsal, feature-unspecific visual input from early visual areas and selected the retinotopic position of the strongest of those inputs. They formed a visual saliency map, with prospective capabilities for rapid scanning, so that a new stimulus was acquired as soon as attention was successfully allocated. Layer $2 / 3$ neurons transformed the phasic signal of layer 4 into an attentional signal at the position of the selected target and stored it until the time of the saccade. They connected to the REC module and activated feature detection and recognition at the currently attended retinotopic position. Hence, they signaled the focus of attention. The REC module in response sent a signal back to layer $2 / 3$ of the FEF when a target was "recognized." "Recognition" in this context meant that the attentional focus could be withdrawn from the current position, either because the target was indeed fully recognized or because no future reward could be expected from that particular position in space. In addition, layer $2 / 3$ neurons drove the motor neurons in layer 5 . (Neurons whose firing was tightly coupled to the motor response will be called "motor neurons," but also correspond to "movement" or "premotor" neurons in the literature.) Therefore, layer $2 / 3$ could be interpreted as both generating an attentional signal and a motor plan.

Layer 5 consisted of two functional types of neurons: "buildup" motor neurons (L5r), which showed ramping activity, and "burst" motor neurons (L5b), which signaled the motor output to the SC and the brainstem. A population of fixation neurons inhibited the ramping activity in layer 5. Layer 6 also had two functional types of neurons: one type (L6a) was driven by layer $2 / 3$ and therefore related to attention, the other (L6s) was excited by the saccadic activity from layer 5b. Neurons in layer 6 projected back to layer 4 and biased the visual selection, or, under some conditions, they excited layer 4 in the absence of a visual input. They provided a "top-down saliency" signal that influenced the visual selection and could even induce a "quasi-visual" signal in layer 4, generated internally and looking like the response to a real visual input (Barash, 2003).

The attention related top-down saliency was selected by a "rule input" from the REC module to layer 6a. In prosaccades and during scanning, layer 6 a did not receive a rule input, in which case the FEF ran in its "default mode" in which the attentional signals in layer 6a were small and did not influence the selection of targets in layer 4 . If the attended target had an antisaccade feature, the rule input targeted all retinotopic positions in layer $6 \mathrm{a}$. This global input allowed layer $6 \mathrm{a}$ to be activated by the layer $2 / 3$ input and hence achieve the remapping required for an antisaccade response. In no-go trials, the rule input specifically targeted the foveal population of layer $6 \mathrm{a}$. The top-down saliency depended on the location currently attended (through layer $6 \mathrm{a}$ as in antisaccade trials), but was also influenced by the last saccade (through layer 6 s), which induced an inhibition-of-return (IR).

Neurons and synapses. The basic elements of the FEF model, IF neurons, and synapses, were defined similarly to those of Salinas (2003). (In our common effort for transparency and reproducibility of computer simulations, our complete code is available at www.ini.uzh.ch/ jakob/ code/FEF_DEMO.zip.) The membrane dynamics of the IF neurons were given by

$$
\tau_{m} \frac{d V(t)}{d t}=-V(t)+g_{e}(t)\left(V(t)-V_{e}\right)+g_{i}(t)\left(V(t)-V_{i}\right) .
$$

The membrane time constant $\tau_{m}$ and the excitatory $V_{e}$ and inhibitory $V_{i}$ reversal potentials are summarized in Table 1 .

The conductances $g_{e}$ and $g_{i}$ consisted of two parts. First, synapses within the FEF were modeled as decaying exponential conductances:

$$
\tau_{e, i} \frac{d g_{e, i}}{d t}=-g_{e, i}
$$

Each spike instantaneously increased the conductance of the corresponding synapse by a fixed weight $g_{e, i} \rightarrow g_{e, i}+w_{e, i}$. Weights and time 
Table 1. Single-neuron parameters

\begin{tabular}{lcc}
\hline & Excitatory neurons & Inhibitory neurons \\
\hline$\tau_{m}$ & $20 \mathrm{~ms}$ & $10 \mathrm{~ms}$ \\
$V_{e}$ & $74 \mathrm{mV}$ & $74 \mathrm{mV}$ \\
$V_{i}$ & $-10 \mathrm{mV}$ & $-10 \mathrm{mV}$ \\
$V_{t h}$ & $20 \mathrm{mV}$ & $20 \mathrm{mV}$ \\
$V_{r}$ & $10 \mathrm{mV}$ & $10 \mathrm{mV}$ \\
$t_{r}$ & $1.8 \mathrm{~ms}$ & $1.2 \mathrm{~ms}$ \\
$g_{m}$ & $25 \mathrm{nS}$ & $20 \mathrm{nS}$ \\
\hline
\end{tabular}

Table 2. Mean values of fluctuating external inputs

\begin{tabular}{lll}
\hline Neuronal population & $\mu_{e}$ & $\mu_{i}$ \\
\hline Layer 4 exc and layer 2/3 exc & 0.472 & 0.34 \\
Layer 4 inh and layer 2/3 inh & 0.46 & 0.40 \\
Layer 5r exc & 0.45 & 0.34 \\
Layer 5r inh & 0.42 & 0.34 \\
Layer 5b exc & 0.38 & 0.30 \\
Layer 5b inh & 0.32 & 0.34 \\
Layer 6a exc & 0.2 & 0.34 \\
Layer 6s exc & 0.44 & 0.34 \\
Fixation neurons & 0.46 & 0.12 \\
Visual input to layer 4 & 0.056 & \\
Fixation input & 0.2 &
\end{tabular}

exc, Excitatory; inh, inhibitory.

constants differed between connections and will be described in a separate paragraph (see Table 3 ).

Second, the external inputs to each neuron were modeled as fluctuating conductances $g_{\text {ext }}(t)$ (Salinas, 2003) and added to the internal conductances:

$$
\tau_{\mathrm{ext}} \frac{d g_{\mathrm{ext}}}{d t}=-\left(g_{\mathrm{ext}}-\mu_{e, i}\right)+\sqrt{D} \chi(t)
$$

The fluctuations of the external input were given by the diffusion constant

$$
D=\sqrt{\frac{\mu_{e, i} w_{e, i}}{\tau_{\mathrm{ext}}}}
$$

and a white Gaussian noise $\chi(t) . \mu_{e, i}$ gave the mean conductance of the external input. The external weights $w_{e}=0.02$ and $w_{i}=0.06$ and the time constant $\tau_{\text {ext }}=3 \mathrm{~ms}$ defined the size and the temporal correlation of the input. Background inputs drove the neurons to spontaneous firing rates of $<10 \mathrm{~Hz}$. Only the fixation neurons had a spontaneous firing rate of $\sim 40 \mathrm{~Hz}$ (Hanes et al., 1998). The $\mu$ values of all the external inputs are given in Table 2.

The visual input to layer 4 was turned on $50 \mathrm{~ms}$ after presentation of the stimulus on the screen, or after the last saccade, and was reduced in intensity to $50 \%$ of the initial value $40 \mathrm{~ms}$ later until it was extinguished or the next saccade was made. This temporal pattern approximated the transient and sustained responses to visual stimulation. The spatial pattern of the visual input was given by the relative strength of the inputs at each retinotopic position. When the population activity of bursting neurons in layer $5 \mathrm{~b}$ crossed a threshold of $50 \mathrm{~Hz}$, it initiated a saccade to the corresponding retinotopic position, and the visual input was updated accordingly.

The fixation input targeted the population of fixation neurons and was turned off $50 \mathrm{~ms}$ after the offset of the fixation stimulus.

Network architecture. The detailed architecture of the FEF local circuit model followed some general principles of cortical architecture. The relative proportion of excitatory and inhibitory neurons reflected the 4:1 ratio observed in cortex. As suggested by experimental data (Douglas et al., 1989; Kritzer and Goldman-Rakic, 1995; Binzegger et al., 2004), the recurrent connections dominated the feedforward connections. The network was then tuned to scan an array of targets and produce single saccades comparable with primate behavior. This manual tuning required the insertion of some additional connections.

The retinotopic structure of the network is indicated in Figure 1. Each layer of the FEF circuit contained several populations of IF neurons located at 21 different retinotopic positions along the horizontal axis. Each retinotopic position in layer 4 and in layer $2 / 3$ contained a population of 100 excitatory and one of 25 inhibitory neurons. In layer 5, populations of 40 excitatory and 25 inhibitory ramping neurons (layer $5 \mathrm{r}$ ) and the same numbers of bursting neurons (layer 5b) were inserted. The number of excitatory neurons per population in layer 5 was reduced so as not to exceed the total number of neurons in layers $2 / 3$ and 4 . However, for stability reasons, the number of inhibitory neurons could not be reduced by the same factor (for a discussion of the strength of inhibition in infragranular layers, see Douglas et al., 1989).

Layer 6 consisted of excitatory populations of 50 attention-related (6a) and 50 saccade-related (6s) neurons at each retinotopic position. Finally, one population of 100 fixation neurons was included in the network. The final ratio of excitatory versus inhibitory neurons within the model resulted to be 3.6:1 (7980:2200), which was close to the desired ratio 4:1.

Figure 2 shows the network with all its connections. Connections are numbered according to Table 3 , and the same numbers in brackets will be used to refer to particular connections in the main text. (For example, [1] is the excitatory connection within layer 4.) The connection between two classes of cells, e.g., excitatory neurons and inhibitory neurons in layer $2 / 3$, was described by

$$
W_{n m, p q}^{\mathrm{I} 23 \mathrm{E} 23}
$$

(the weight of the synapse from neuron $m$ in the excitatory population $q$ of layer $2 / 3$ to neuron $n$ in the inhibitory population $p$ of layer 2/3). The individual synaptic weights were assigned as follows.

A population weight matrix

$$
W_{p q}^{\mathrm{I} 23 \mathrm{E} 23}
$$

defined the average weight of the synapses between population $q$ in layer 4 and population $p$ in layer $2 / 3$. Individual weights were randomly distributed between 0.5 and 1.5 times this average weight. The connectivity between two populations was made $50 \%$ by randomly setting half of the weights to zero (Fig. 2, bottom). In the excitatory to inhibitory connections within layers 4 [2] and $2 / 3$ [8], $75 \%$ of the weights were set to zero, resulting in $25 \%$ connectivity. This randomness in the connection between two populations ensured that the inputs to single neurons differed. The average weights and time constants of all connections are listed in Table 3.

There were three major classes of connections: local, global, and special purpose. Local connections (Fig. 2, solid lines) were described by the weight matrix

$$
W_{p q}^{A B}=w^{A B} \delta_{p q},
$$

with $\delta_{p q}=1$ if $p=q$ and 0 otherwise. The self-excitation within layer 4 [1] included a weak nearest neighbor interaction:

$$
W_{p q}^{\mathrm{E} 4 \mathrm{E} 4}=w^{\mathrm{E} 4 \mathrm{E} 4}\left(\delta_{p q}+0.05\left(\delta_{p(q-1)}+\delta_{p(q+1)}\right)\right) .
$$

The connection from layer $2 / 3$ excited inhibitory neurons in layer 4 locally and included nearest neighbors [6]:

$$
W_{p q}^{14 \mathrm{E} 23}=w^{14 \mathrm{E} 23}\left(\delta_{p q}+\delta_{p(q-1)}+\delta_{p(q+1)}\right) .
$$

Global connections (Fig. 2, dashed lines) targeted all retinotopic positions. These connections were fully described by their weight

$$
W_{p q}^{A B}=w^{A B} .
$$

Finally, some connections were more specific than the local and global ones described above. Such special-purpose connections (Fig. 2, dashdotted lines) were required for the remapping of visual activity in the anti-saccade task or to provide an inhibition-of-return.

The connection from layer $6 \mathrm{~s}$ neurons to inhibitory neurons in layer 4 [5] consisted of two components: a global fast component with weight $w^{\text {I4E6s }}$ that reset the activity in layer 4 after each saccade and a slow 
component ( $\tau=50 \mathrm{~ms}$ ) of excitation to inhibitory neurons that represented the position mirrored at the vertical meridian:

$$
W_{\mathrm{IR}, p q}^{\mathrm{I}, \mathrm{E} 6 \mathrm{~s}}=w_{\mathrm{IR}}^{\mathrm{I} 4 \mathrm{E} 6 s} \delta_{p(2 z-q)} .
$$

Here $z$ is the position of the fovea relative to the leftmost position represented in the network. In the retinotopic coordinates of the model, this mirrored position of a target corresponds to where the current location of the fovea will be after the saccade to the target. Hence, this connection introduced an inhibition-of-return in the visual selection process of layer 4 attributable to an inhibition of activity at the retinotopic location of the last-foveated target.

The connection from layer 6 a to layer 4 excitatory neurons [4] provided the antisaccade rule in the network, i.e., the visual target opposite to the currently attended location should be selected next. The connection matrix was given by

$$
W_{p q}^{\mathrm{E} 4 \mathrm{E} 6 \mathrm{a}}=w^{\mathrm{E} 4 \mathrm{E} 6 \mathrm{a}} \delta_{p(2 z-q)} .
$$

Excitatory neurons in layer $5 b$ globally excited all populations of inhibitory neurons in layer $2 / 3$ [11] except for the foveal one:

$$
W_{p q}^{\mathrm{I} 23 \mathrm{E} 6 \mathrm{a}}=w^{\mathrm{I} 23 \mathrm{E} 6 \mathrm{a}}\left(1-\delta_{z p}\right) .
$$

The feedback connection from excitatory neurons of layer $5 b$ to excitatory neurons in layer $2 / 3$ [12] targeted the foveal representation only:

$$
W_{p q}^{\mathrm{E} 23 \mathrm{E} 5 \mathrm{~b}}=w^{\mathrm{E} 23 \mathrm{E} 5 \mathrm{~b}} \delta_{z p}
$$

This connection reset the attentional activity in layer $2 / 3$ back to the fovea after each saccade.

Finally, the fixation neurons received excitatory input from the foveal representation in layer $2 / 3$ [24] and were inhibited by the inhibitory neurons in layer $5 \mathrm{r}$ [25]:

$$
W_{q}^{\mathrm{IFIX} \mathrm{E23}}=w^{\mathrm{IFIX} \mathrm{E23}} \delta_{q z} \text { and } W_{q}^{\mathrm{IFIXI5r}}=w^{\mathrm{IFIXI5r}} .
$$

The fixation neurons prevented the buildup of motor activity by inhibiting all retinotopic positions in layer 5r [17]:

$$
W_{p}^{\mathrm{E} 5 \mathrm{rIFIX}}=w^{\mathrm{E} 5 \mathrm{rIFIX}} .
$$

To compare the connectivity of the FEF model circuit to the connectivity matrix for cat visual cortex in the study by Binzegger et al. (2004), the strength of each connections was calculated as the product of the average synaptic weight, the synaptic time constant, and the number of synapses in the connection:

$$
s^{A B}=w^{A B} \tau^{A B} N^{A B} .
$$

This product provided a direct measure of the strength of a connection. The values of the strengths of all connections are listed in Table 3. Only two connections ([5] and [11]) deviated strongly from the connectivity pattern in cat visual cortex. Both of them controlled the activity of the network after saccades.

Implementation of the recognition module and mapping to rule. The recognition (REC) module consisted of three arrays of feature detectors for

\section{Network layout}

Dorsal

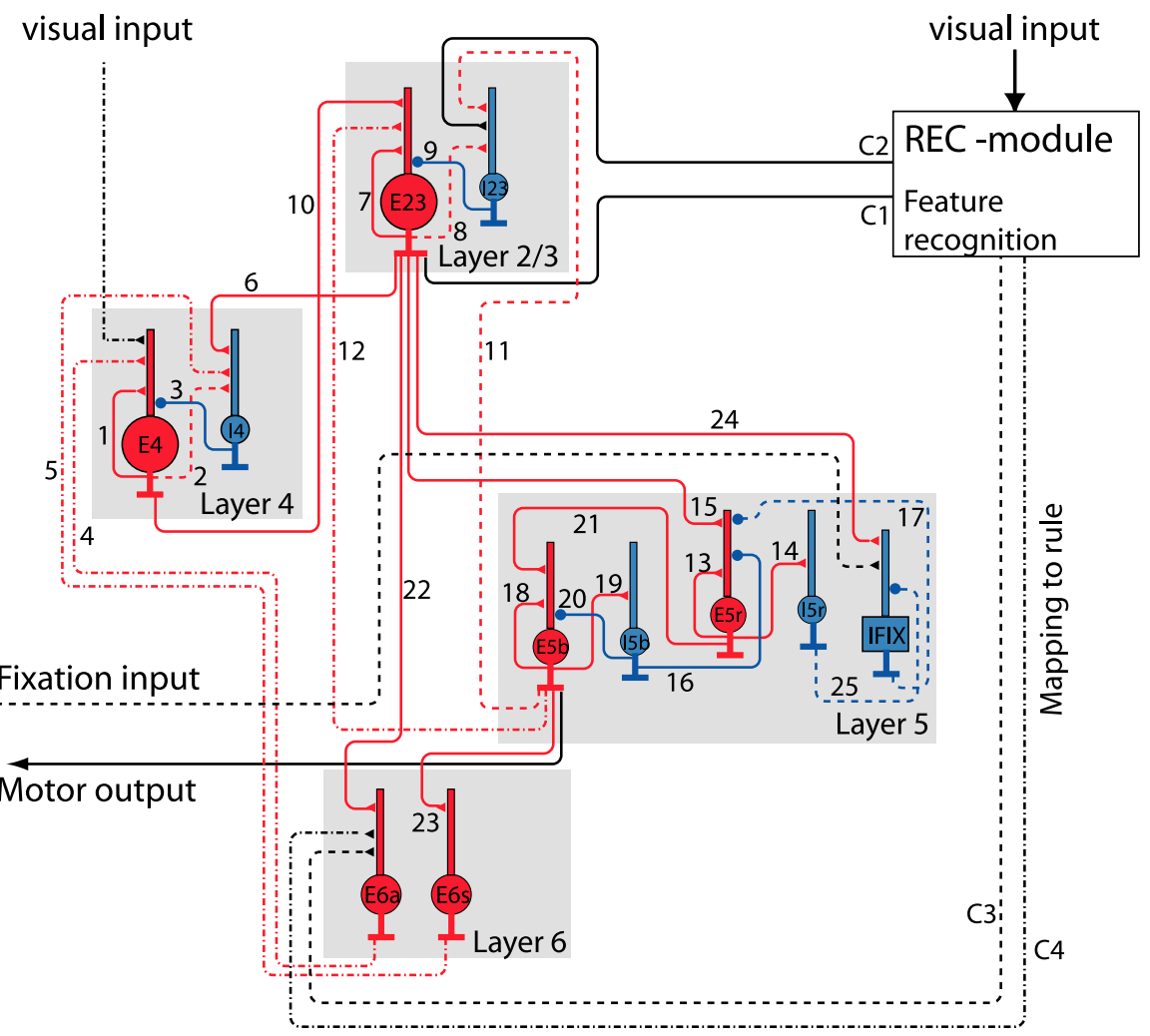

Connections within a layer
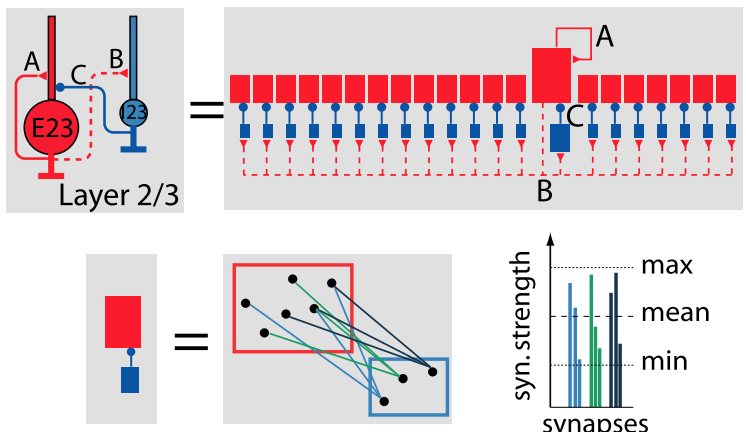

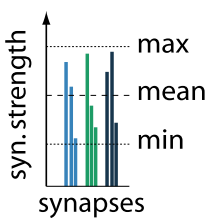

Legend

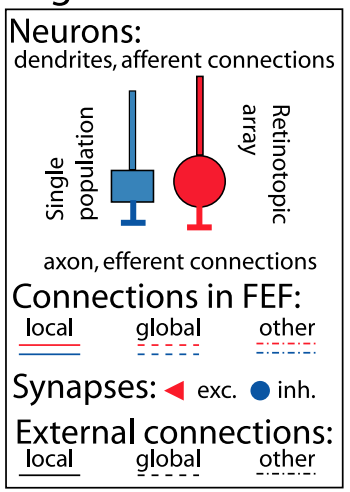

Figure 2. Layout of the FEF circuit. Top, Complete network architecture. Colored circles are full retinotopic representations consisting of arrays of 21 populations of neurons. Colored rectangles are single populations, e.g., fixation neurons (red, excitatory; blue, inhibitory). Layer 4 received a visual input from the dorsal stream, which is not feature specific. The fixation neurons received a fixation input, and the motor output of the FEF was given by the bursting neurons in layer 5 of the FEF. The spatial pattern of the connections is summarized into three groups: local connections (solid lines) connected only to populations at the same retinotopic position, global connections (dashed lines) connected to all retinotopic positions, and the connections that could not be grouped into one of the two above, which were called other connections (dash-dotted line). All connections are numbered according to Table 3. External inputs and the connections to and from the REC module are shown in black. The REC module received a feature-specific visual input, which represented the ventral processing stream. Layer $2 / 3$ connected to the REC module [C1] and in turn received input from it [C2]. Layer 6a of the FEF received the rule input directly from antisaccade [C3] and no-go [C4] feature detectors in the REC module. A detailed description of the REC module and its interactions with the FEF is given in the supplemental data (available at www.jneurosci.org as supplemental material). Bottom left, Retinotopic arrangement of the connections in layer 2/3. A, Local self-excitation (only shown for 1 retinotopic position). B, Global excitation of all inhibitory populations (only shown for 1 efferent excitatory population). C, Local inhibitory connections. Bottom, lllustration of the random selection of connections for three sample inhibitory neurons connecting randomly to $50 \%$ of the excitatory neurons. The distribution of the weights is indicated by the histogram on the right. The minimum, maximum, and mean of the uniform distribution are shown by the horizontal dashed lines. 
Table 3. Parameters of connections within the local circuit model

\begin{tabular}{|c|c|c|c|c|c|c|c|}
\hline Connection & To & From & Type & Weight & $\tau(\mathrm{ms})$ & Strength & Fig. 2 \\
\hline$W_{p q}^{\mathrm{E4} \mathrm{E}}$ & L4 exc & L4 exc & 1 & 0.016 & 5 & 9200 & 1 \\
\hline$W_{p q}^{14}$ & L4 inh & L4 exc & g & 0.01 & 5 & 13781 & 2 \\
\hline$W_{p q}^{\mathrm{E}} 4 \mathrm{q}$ & L4 exc & L4 inh & I & 0.12 & 3 & 9450 & 3 \\
\hline$W_{p q}^{\mathrm{E} 4 \mathrm{E} 6 \mathrm{a}}$ & L4 exc & L6a exc & 0 & 0.008 & 5 & 2100 & 4 \\
\hline$W_{p q}^{14 \mathrm{E} 6 \mathrm{~s}}$ & L4 inh & L6s exc & $g$ & 0.008 & 10 & 22050 & 5 \\
\hline$W_{l R, p q}^{14}$ & L4 inh & L6s exc & 0 & 0.0016 & 50 & 1050 & 5 \\
\hline$W_{p q}^{14,23}$ & L4 inh & $L 2 / 3$ exc & I & 0.0028 & 5 & 1068 & 6 \\
\hline$W_{p q}^{\mathrm{E} 23 \mathrm{E} 23}$ & $L 2 / 3$ exc & $L 2 / 3$ exc & I & 0.0096 & 10 & 10080 & 7 \\
\hline$W_{p q}^{123 \mathrm{E} 23}$ & $\mathrm{~L} 2 / 3 \mathrm{inh}$ & $L 2 / 3$ exc & g & 0.008 & 5 & 11025 & 8 \\
\hline$W_{p q}^{\mathrm{E} 23123}$ & $L 2 / 3$ exc & $\mathrm{L} 2 / 3 \mathrm{inh}$ & I & 0.16 & 3 & 12600 & 9 \\
\hline$W_{p q}^{\mathrm{E} 23 \mathrm{E} 4}$ & L2/3 exc & L4 exc & I & 0.0032 & 5 & 1680 & 10 \\
\hline$W_{p q}^{123}$ E5b & L2/3 inh & L5b exc & 0 & 0.04 & 5 & 42000 & 11 \\
\hline$W_{p q}^{\mathrm{E} 23 \mathrm{E}}$ & L2/3 exc & L5b exc & 0 & 0.017 & 10 & 7140 & 12 \\
\hline$W_{p q}^{\mathrm{Esr} E 5 \mathrm{r}}$ & L5r exc & L5r exc & I & 0.004 & 50 & 3360 & 13 \\
\hline$W_{p q}^{19 \mathrm{rES}}$ & L5rinh & L5r exc & I & 0.03 & 5 & 1575 & 14 \\
\hline$W_{p q}^{E E \text { ESE }}$ & L5r exc & $L 2 / 3$ exc & I & 0.0026 & 5 & 528 & 15 \\
\hline$W_{p q}^{E-5 r}$ & L5r exc & L5b inh & I & 0.04 & 10 & 4200 & 16 \\
\hline$W_{p q}^{\mathrm{ESI} I F \mid X}$ & L5r exc & Fix. inh & g & 0.007 & 3 & 882 & 17 \\
\hline$W_{p q}^{E 5 b \text { E5b }}$ & L5b exc & L5b exc & I & 0.12 & 5 & 10080 & 18 \\
\hline$W_{p q}^{15 \mathrm{~b} \mathrm{E5b}}$ & L5b inh & L5b exc & I & 0.1 & 5 & 5250 & 19 \\
\hline$W_{p q}^{E 5 b}$ & L5b exc & L5b inh & I & 0.25 & 3 & 7875 & 20 \\
\hline$W_{p q}^{E 5 b E 5 r}$ & L5b exc & L5r exc & I & 0.02 & 5 & 1680 & 21 \\
\hline$W_{p q}^{\text {EGa E23 }}$ & L6a exc & $\mathrm{L} 2 / 3 \mathrm{exc}$ & I & 0.01 & 5 & 2500 & 22 \\
\hline$W_{p q}^{\mathrm{E} q \mathrm{q} \text { E E5b }}$ & L6s exc & L5b exc & I & 0.08 & 5 & 8400 & 23 \\
\hline$W_{p q}^{|F| F \mid X ~ E 23 ~}$ & Fix. inh & L2/3 exc & I & 0.004 & 5 & 100 & 24 \\
\hline$W_{p q}^{1+f|X|} 15 r$ & Fix. inh & L5rinh & 0 & 0.1 & 3 & 7875 & 25 \\
\hline
\end{tabular}

exc, Excitatory; inh, inhibitory; g, global connection; I, local connection; 0, other connection. The column Fig. 2 indicates the number of the connection in Figure 2.

the three features (prosaccade, antisaccade, and no-go) and an array of neurons expressing the recognition of the target. The feature detectors received a feature specific visual input, which simulated the input from the ventral processing pathways. Riesenhuber and Poggio (1999) provided a possible implementation of such feature detectors in a neural network. FEF also received a feature-independent input from the dorsal pathway. Figure $3 A$ outlines the REC module and its connections to the FEF. By a retinotopically specific release of inhibition, the attentional activity in layer $2 / 3$ directly selected the retinotopic position at which the feature detectors should respond [C1]. All other retinotopic positions were suppressed. Hence, the feature detectors responded with a firing rate of $\sim 70 \mathrm{~Hz}$ only if their preferred feature was at the attended location.

The feature detectors projected to layer $6 \mathrm{a}$ and directly provided the rule input that depended on the feature of the visual input that was being attended to currently. This occurred in the following way: prosaccade features did not influence the FEF, but antisaccade feature detectors excited all retinotopic positions in layer $6 \mathrm{a}$ [C3]. This excitation allowed layer $2 / 3$ of the FEF to drive neurons in layer $6 \mathrm{a}$. By this means, the remapping of the visual stimulus, as given by the connection from layer 6 a to layer 4 , was activated. It is important to note that the input from the antisaccade feature detectors was not strong enough to drive layer 6 a. However, in conjunction with the global rule input, the attention specific input from layer $2 / 3$ was able to drive layer 6 a neurons representing the retinotopic position currently attended. This activation was then remapped onto visual neurons in layer 4 through the direct connection from layer $6 \mathrm{a}$. The no-go feature detectors, conversely, excited only the foveal population of layer 6a [C4] and induced a remapping of visual activity to the fovea. This input was strong enough to directly elicit activity in layer 6a.

The actual recognition neurons were driven locally by the feature detectors. Prosaccade features drove recognition only at the fovea, whereas antisaccade and no-go features were recognized at any retinotopic position. The recognition population was modeled similarly to layer 5 in the FEF. A population of ramping neurons introduced a delay between the onset of activity of the feature detectors and the burst of activity that

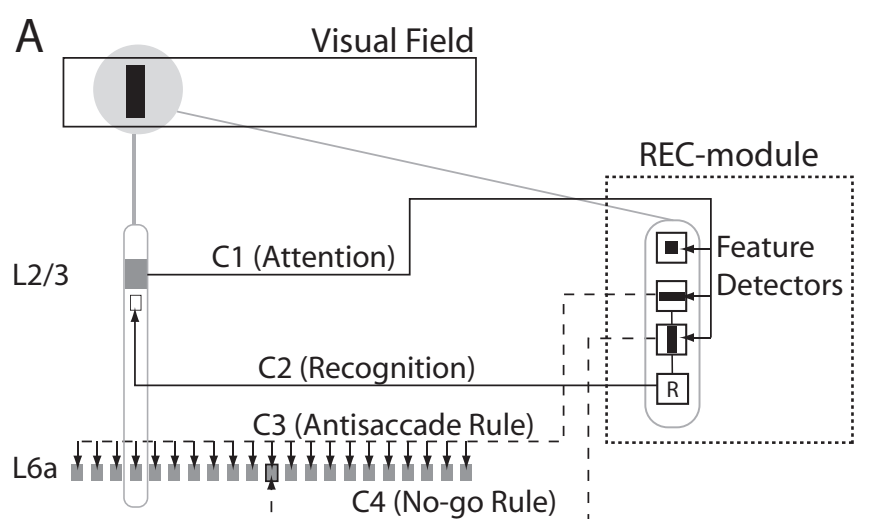

B

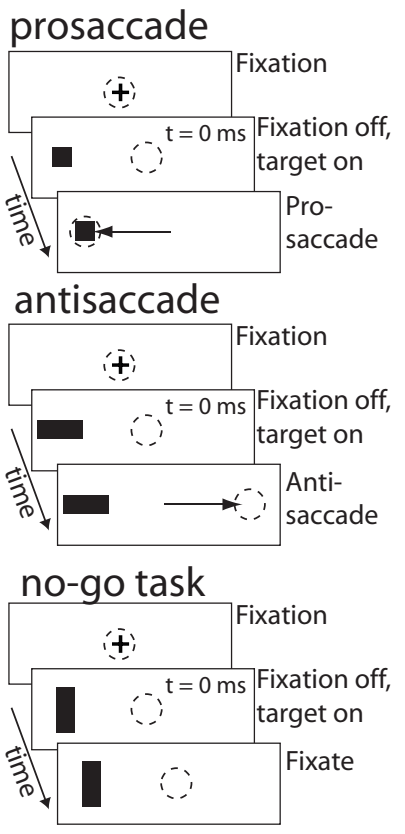

delayed memory saccade

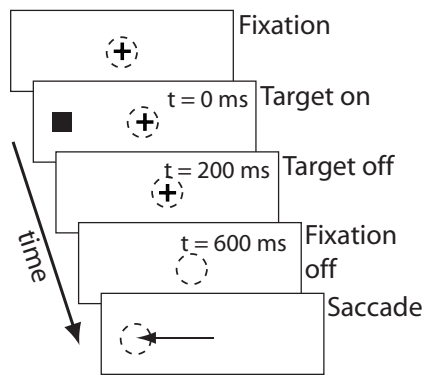

Figure 3. Connections of the FEF with the REC module and saccade tasks. A, Feature detection and mapping to the rule. Visual input directly drove feature detectors in the REC module. The attentional input from layer $2 / 3$ of the FEF selected the retinotopic position at which the REC module could respond. All retinotopic positions outside the focus of attention (not shown in the figure) were suppressed. Antisaccade feature detectors directly excited all populations in layer 6a of the FEF. No-go feature detectors connected to the foveal position in layer 6a (central population with black border). In addition, antisaccade and no-go feature detectors projected to a recognition population. The recognition was modeled as a buildup of neuronal activity that led to a burst of activity, similar to that of layer 5 of the FEF. The bursting recognition neurons projected to inhibitory neurons in layer $2 / 3$ at the same retinotopic position (white box). $\boldsymbol{B}$, Sequences of visual inputs for different tasks. Time always runs from top left to bottom right. Crosses indicate fixation points. Dashed circles show the desired position of gaze for a correct trial. In all tasks, time was aligned to the onset of the visual stimulus. The fixation point always appeared before that point in time without any specific delay. In all single saccade tasks, the visual input was shown for $200 \mathrm{~ms}$. In the delayed memory saccade task, the fixation input was turned off at $t=600 \mathrm{~ms}$, whereas in all other tasks, it was turned off at time $0 \mathrm{~ms}$. For the scanning task, the network was presented an array of six targets with different stimulus intensities. It freely scanned the array for $60 \mathrm{~s}$.

signaled the recognition of a target. The burst of recognition excited inhibitory neurons in layer $2 / 3$ and turned off the memory and attentionrelated activity in layer $2 / 3$. Hence, the recognition signal corresponded to the command to release attention. A detailed description of the REC module and its connections to the FEF is given in the supplemental data (available at www.jneurosci.org as supplemental material).

Behavioral tests and simulations. The behavior of the network was tested in different tasks (Fig. $3 B$ ). First, the behavior of the network was 
assessed for visual saccades and delayed memory saccades. In these tasks, the network "fixated" on a point. A visual stimulus at a single retinotopic position was turned on at time $0 \mathrm{~ms}$ and was kept on for $200 \mathrm{~ms}$. Simultaneously the fixation input was turned off. The network immediately made a saccade to the stimulus. In the delayed memory saccade task, the fixation point was turned off $600 \mathrm{~ms}$ after stimulus presentation. The stimulus was presented for $200 \mathrm{~ms}$ as in the visual saccade task.

Second, the network was tested with a task in which the network had to select one of three responses (prosaccade, antisaccade, or no-go), depending on the nature of the target. The recognition of the target shape and the corresponding rule input were given by the REC module. As an additional demonstration of the remapping, the network was tested in a delayed memory antisaccade task.

Third, we ran the network while freely scanning an array of six targets, which differed in their intensity. The six targets had the relative strengths 0.9:1:0.8:1:0.9:0.8. In this last case, the task of the network was to freely look around in the visual scene for $60 \mathrm{~s}$. This paradigm, which illustrated the effect of the inhibition-of-return, was simulated with five different versions of the network.

All simulations were run in Matlab (MathWorks, Natick, MA) using a first order Euler method with integration time steps of $0.1 \mathrm{~ms}$. Test simulations with a shorter time step of $0.01 \mathrm{~ms}$ did not reveal any significant changes in the results.

Data analysis. The spiking of the populations of all neurons within the network was saved for each simulation. Most results will be reported as population activities. The number of spikes within a population was counted in time bins of $1 \mathrm{~ms}$ and then smoothed by a synaptic kernel (Sato and Schall, 2003):

$$
S(t)=\frac{\left(1-\exp \left(-\frac{t}{\tau_{\text {rise }}}\right)\right) \exp \left(-\frac{t}{\tau_{\text {decay }}}\right)}{\int_{0}^{\infty}\left(1-\exp \left(-\frac{t^{\prime}}{\tau_{\text {rise }}}\right)\right) \exp \left(-\frac{t^{\prime}}{\tau_{\text {decay }}}\right) d t^{\prime}}
$$

$\left(\tau_{\text {rise }}=1 \mathrm{~ms}, \tau_{\text {decay }}=10 \mathrm{~ms}\right)$. In the delayed memory saccade task, responses of single neurons were averaged over all correct saccades and binned into time windows of $1 \mathrm{~ms}$. Traces were aligned with the temporal onset to show visual activity and to the time of the saccade for movement activity. Again the result was smoothed by the synaptic kernel.

The behavioral data were given by the activity in the bursting motor neurons in layer $5 b$, which signaled both the location and the timing of saccades. Reaction or fixation times were binned in time windows of 10 $\mathrm{ms}$ and are shown as histograms. Average values are always reported as mean $\pm \mathrm{SD}$.

\section{Results}

The local circuit model of the FEF simulated several different tasks. First, it was used to control eye movements in visual and delayed memory saccades. In these tasks, a single visual target was presented in the periphery, and the network had to make a saccade to it as quickly as possible in the visual saccade task and after a delay in the memory saccade task. The responses of populations of neurons and single cells in these two tasks are presented in the figures to illustrate how the circuit transformed the visual input to an oculomotor output and to enable a comparison of the network activity with results from equivalent experiments in awake behaving monkeys. Throughout the paper, exact references to experimental papers will be given, citing the figures that correspond to the simulation results.

Second, the network performed a saccade versus antisaccade task. According to the shape of the visual target, the saccade had to be made toward that target (prosaccade trial) or away from it (antisaccade trial) or fixation had to be maintained (no-go trial). This second task showed how the FEF circuit could use a topdown rule to select a particular strategy for its eye movements. The behavior of the network in this case was switched between
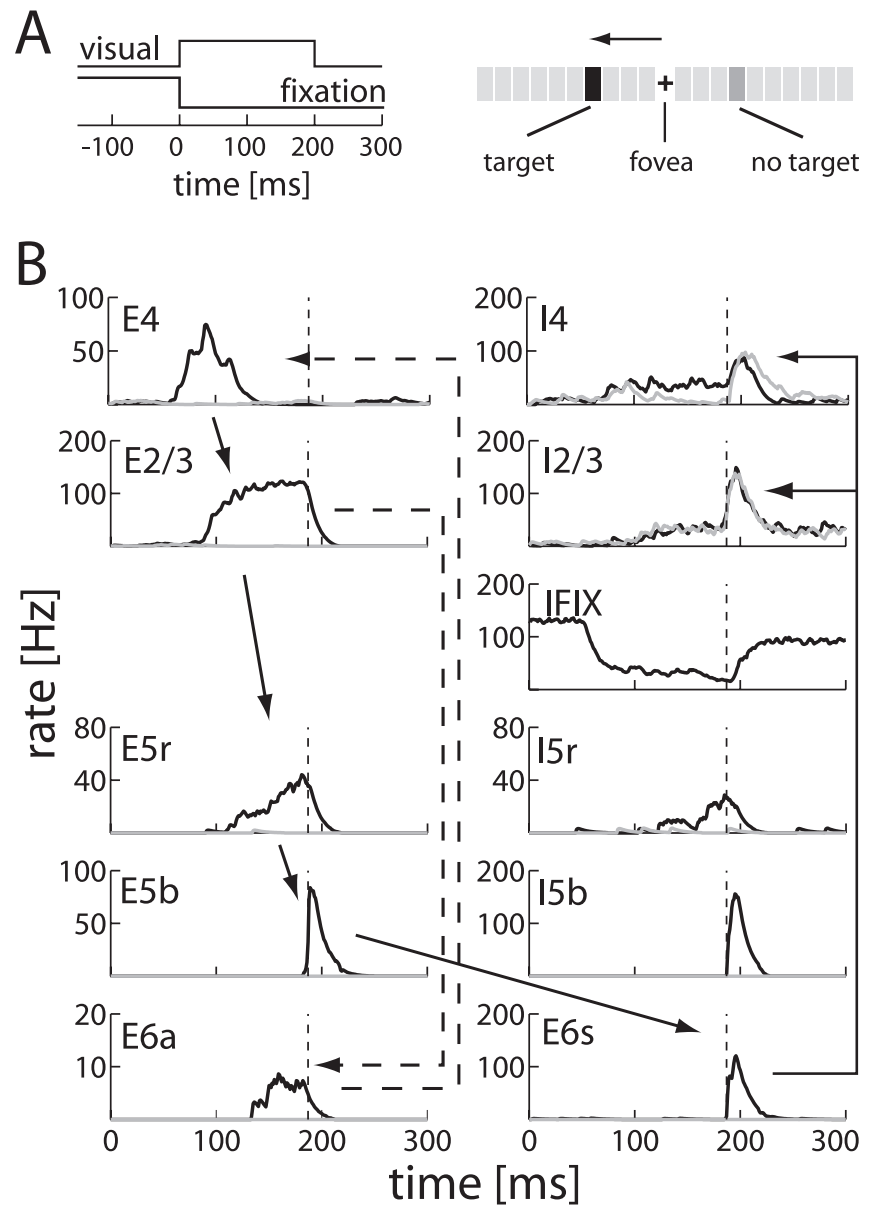

Figure 4. Visual saccade. $\boldsymbol{A}$, Left, Timing of external inputs in the saccade task. Fixation input was turned off simultaneously with the visual target onset. Right, Spatial arrangement of populations shown in the graphs below. $\boldsymbol{B}$, The population rates for two selected retinotopic positions are shown for all layers of the FEF. Black traces, Populations representing the location of the target; gray traces, populations representing the location mirrored at the vertical meridian (see $\boldsymbol{A}$ ). The type of the neuronal populations (excitatory or inhibitory) and the layered position are indicated by the insets. Filled arrows show the flow of information along the feedforward pathway (compare with Fig. 1). The dashed arrows are the connections involved in biasing the visual selection according to the location being currently attended. Note that the inhibitory neurons in layers 4 and 2/3 are not tuned to a specific direction. They fire a postsaccadic burst that is involved in resetting activity in those two layers.

strategies by the input from the REC module, whereas the structure of the FEF network remained exactly the same.

Third, the network scanned an array of inputs. This scanning paradigm was used to test how well the model behaved under the condition of a constant visual input. The results of all simulations were traces of population activity or single-cell firing, together with the behavioral data of the eye movement traces.

\section{Visual saccades}

In the visual saccade task (Fig. 4), the peripheral stimulus was presented to the network at time $0 \mathrm{~ms}$ and lasted for $200 \mathrm{~ms}$. Most neurons had spatially tuned activity as indicated by the higher firing rates for neurons representing the target position (Fig. $4 B$, black traces) compared with neurons at the retinotopic position mirrored at the vertical meridian (Fig. $4 B$, gray traces).

Excitatory neurons in layer 4 responded with a phasic visual activity with a latency of 50-100 ms after stimulus presentation. Their activity was transmitted to excitatory neurons in layer $2 / 3$, and they in turn were suppressed by inhibitory neurons in layer 4 
A
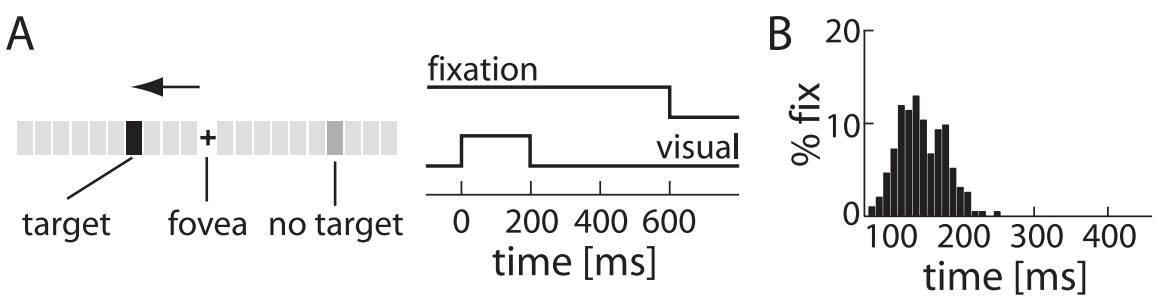

C

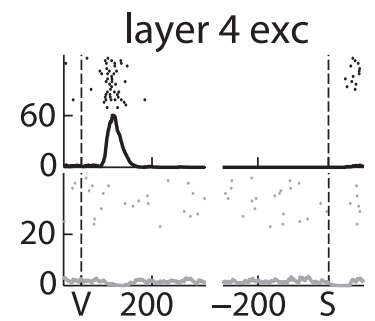

layer 4 inh
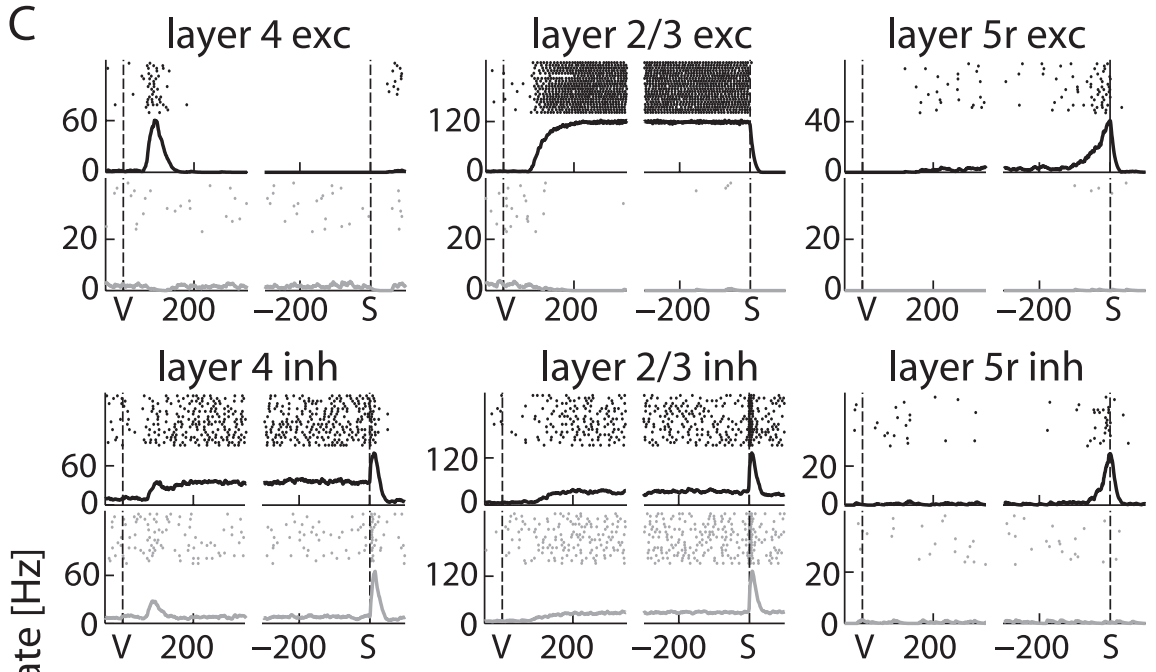

layer $2 / 3$ inh
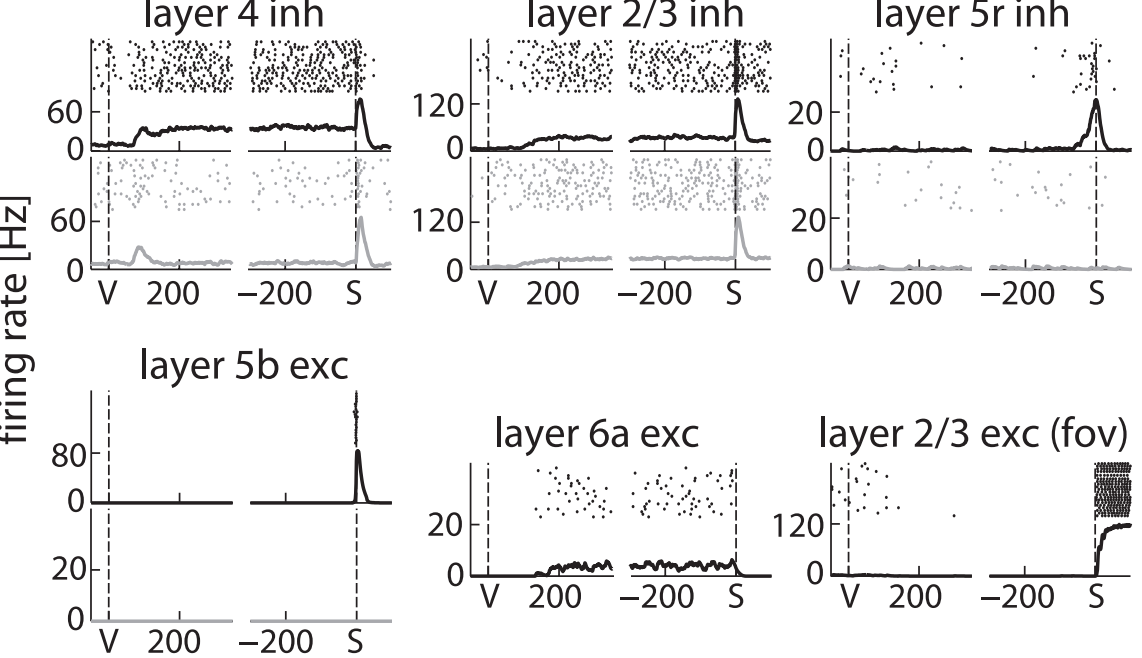

layer $2 / 3$ exc (fov)

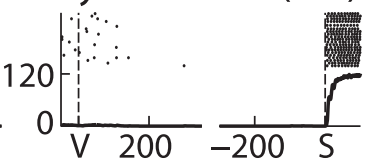

layer $5 b$ inh

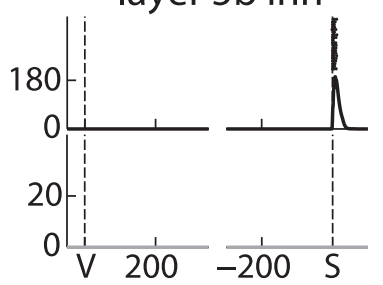

layer 6s exc
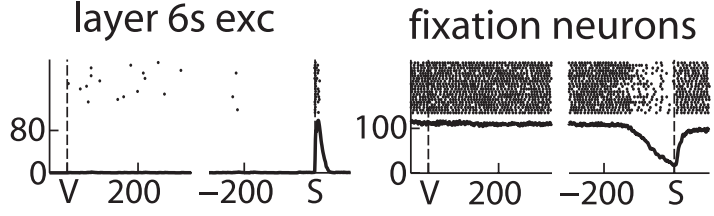

time $[\mathrm{ms}]$

Figure 5. Responses of single neurons in the delayed memory saccade task. $A$, Temporal and spatial characteristics of the visual input. Left, The retinotopic organization of the FEF model is shown with the fixation point at the fovea (black cross), the target position (black square), and a randomly chosen position different from the target (dark gray square). Right, Temporal pattern of visual inputs to the network. $\boldsymbol{B}$, Distribution of reaction times after fixation point offset (mean $\pm S D, 147 \pm 32 \mathrm{~ms}$ ). $\boldsymbol{C}$, Responses of single cells for all cell classes of the network. The firing of single neurons is shown as spike raster plot (20 random samples) and the average firing rate over all correct trials $(n=193)$. Responses are aligned to the visual stimulus ( $V$, left dashed vertical line) and to the saccade onset (S, right dashed vertical line) for each neuron. Black traces and raster plots correspond to neurons that have their response field at the position of the visual target, and gray traces and rasters are neurons with a different response field. exc, Excitatory; inh, inhibitory.

driven by feedback from layer $2 / 3$. This resulted in a phasic visual activity of layer 4 neurons, which was suppressed already before the upcoming saccade [Bruce and Goldberg (1985), their Fig. 3], whereas neurons in layer $2 / 3$ fired until the time of the saccade [Bruce and Goldberg (1985), their Fig. 4]. The firing in layer 2/3 drove the ramping neurons in layer $5(5 \mathrm{r})$, which increased their firing until the time of saccade [Bruce and Goldberg (1985), their
Fig. 5]. The ramping had the effect of delaying the motor output compared with the onset of visual selection and attention in layers 4 and $2 / 3$. Fixation neurons were suppressed by layer $5 \mathrm{r}$ activation and had enhanced firing when the fixation input was on [Hanes et al. (1998), their Fig. 8]. Sufficiently high firing of the excitatory layer $5 r$ neurons drove a burst in layer $5 b$ that initiated the saccade [Segraves (1992), his Fig. 5]. The resetting of the activity of the network after the saccade occurred through the inhibitory neurons in layers 4 and $2 / 3$. These neurons responded with a burst of activity at each saccade regardless of the target location. Note that, with exception of the inhibitory neurons in layers 4 and $2 / 3$, all neurons were tuned to their retinotopic position.

The network activity was simulated 200 times for the same visual saccade task. The average reaction time over all trials was $246 \pm 33 \mathrm{~ms}$ (for the distribution of reaction times, see Fig. 7).

\section{Delayed memory saccades}

In the delayed memory saccade task (Fig. $5 A$ ), the saccade to the position of the peripheral target could only be made after a delay during which the target disappeared, which meant that the position of the target had to be remembered by the network. The presence of a fixation command was modeled as a constant input to the fixation neurons up to $t=650 \mathrm{~ms}$. The elevated activity of the fixation neurons suppressed the build up of activity in layer 5r "ramping" neurons. The network made $96.5 \%$ (193 of 200) saccades to the target with an average reaction time of $147 \pm 32 \mathrm{~ms}$ after fixation point offset (Fig. $5 B$ ), which was faster than reported in experiments (Roesch and Olson, 2005). In this task, the main delay was the time to build up activity in layer 5, whereas in the case of visual saccades, additional time was required to make the visual selection.

Figure $5 C$ shows the single-cell responses of all classes of neurons within the network. Neurons with their receptive field at the position of the memory target were compared with neurons that had a different receptive field position. These single-cell responses were directly comparable with single-cell measurements in awake behaving monkeys.

Excitatory neurons in layer 4 were visual neurons showing a transient response to a visual input [Hanes et al. (1998), their Fig. $4 a$ ]. The winner-take-all competition between these neurons permitted only one population to respond maximally to the visual input. Only one retinotopic position was therefore selected. Inhibitory neurons in layer 4 had directionally tuned responses during the delay period because of the feedback from layer $2 / 3$. At 
the time of each saccade, they showed a phasic, spatially unspecific response. Excitatory neurons in layer $2 / 3$ provided the short-term memory of the circuit. Recurrent excitation allowed a population of neurons to sustain their activity at the selected retinotopic position until the saccade was made [Umeno and Goldberg (2001), their Fig. 9; Thompson et al. (2005a), their Fig. 3d]. Inhibitory neurons had untuned delay activity and responded with a burst after each saccade. The global bursting of inhibitory neurons suppressed prospective visual activity in layer 4 and short-term memory in layer $2 / 3$ after saccades to allow the FEF to process the "new" visual input whose retinotopy was changed according to the saccade.

The two classes of layer 5 cells showed spatially selective motor responses. Ramping neurons ( $5 r$ ) showed an activity that increased toward the time of the saccade, and "bursting" neurons (5b) exhibited a clear burst of activity for each saccade. This saccadic burst at a particular retinotopic position constitutes a clear temporal and spatial signal for the SC and motor neurons in the brainstem [Segraves (1992), his Fig. 5]. Neurons in layer 6 showed activity related to attention (layer 6a) and related to the saccadic burst (layer 6s).

A special group of neurons represented the fovea. An elevated activity of foveal neurons in layer $2 / 3$ signaled that the focus of attention was on the foveal representation. The excitatory feedback from layer $5 b$ ensured that this foveal attention was activated after each saccade. Fixation neurons responded strongly while the external fixation input was turned on. Their activity was suppressed toward the saccade attributable to input from layer $5 \mathrm{r}$ inhibitory neurons [Segraves (1992), his Fig. 8]. After each saccade, the excitatory input from foveal neurons in layer $2 / 3$ excited the fixation neurons.

\section{Rule-dependent remapping of visual inputs}

The excitatory connection from layer 6a to layer 4 enabled the network to influence the selection of the next target according to the currently attended location. This top-down bias of selection, however, was only effective if the firing rate of neurons in layer $6 \mathrm{a}$ was enhanced by a global excitatory rule input. In our model, this rule input was feature dependent and was given by the input from antisaccade feature detectors in the REC module to layer 6 a of the FEF. It is important to notice that this rule input did not have any spatial content or preference, but rather enabled the FEF circuit to use the attentional signal to influence the next visual selection. The possibility of influencing the visual selection with respect to the location being currently attended to, or even to produce a quasi-visual, internally generated neuronal signal that looked like the response to a real visual input (Barash, 2003), allowed the network to control eye movements according to specific rules. Primates can use such a rule in a prosaccade versus antisaccade task. The network was simulated in a task similar to one studied in primates (Sato and Schall, 2003), in which the shape of the visual target defined whether the network had to make a prosaccade or an antisaccade or hold fixation in a no-go trial.

The remapping of activity in the antisaccade and no-go tasks will be described below. The behavior of the network and the firing of some selected groups of neurons were compared for the different tasks.

As in a normal visual saccade, layer 4 always selected the visual target and layer $2 / 3$ signaled the attentional focus on the target (Fig. 6A, $B$ ). If the network attended an antisaccade feature stimulus, the corresponding populations of feature detectors in the REC module responded. The input from the antisaccade feature detectors increased the activity in all populations of layer $6 \mathrm{a}$. In particular, the input from layer $2 / 3$ resulted in a higher firing rate of the layer 6 a neurons compared with their firing in the visual saccade task (compare with Fig. $4 B$ ). This resulted in a top-down visual signal in layer 4 at the prospective landing position of the saccade, i.e., opposite to the visual stimulus [observed in the lateral intraparietal area (LIP) by Zhang and Barash (2000), their Fig. 4]. In addition to this remapping, the recognition signal (Fig. $6 A$, bottom right) excited inhibitory neurons in layer $2 / 3$ and, hence, suppressed activity in layer $2 / 3$. The recognition signal was 
necessary to shift the focus of attention in layer $2 / 3$ away from the visual target. The enhanced firing of inhibitory neurons in layer $2 / 3$ after the saccade was required to reset the network activity.

In the no-go task, the rule input from no-go feature detectors in the REC module targeted only the foveal population, which resulted in a quasi-visual signal and a shift of attention back to the fovea (Fig. $6 B$ ). Again, the recognition signal allowed the shift of attention to occur. Without it, layer $2 / 3$ excitatory neurons would stay active until a saccade was made to the position of the visual input.

The network was run 200 times for each of the three conditions of the task (Fig. 3B). Prosaccades corresponded exactly to the visual saccade trials explained previously. All 200 saccades were made correctly to the visual target. The average reaction times of $246 \pm 33 \mathrm{~ms}$ for the saccades (Fig. 7A) were slower than those observed in monkey experiments (Amador et al., 1998; Everling et al., 1999).

In the antisaccade task, the network made 92\% (184 of 200) correct responses with a reaction time of $346 \pm 29 \mathrm{~ms}$ (Fig. 7A). Again, these reaction times were slower than in monkey experiments (Amador et al., 1998; Everling et al., 1999). The errors in the antisaccade task were either erroneous prosaccades ( 3 of 200) or no saccade was made within $450 \mathrm{~ms}$ after stimulus presentation (13 of 200). In the no-go task, the network always successfully suppressed the saccade.

Figure $7 B$ shows the firing pattern of four different types of neurons and compares their activation pattern for prosaccades and antisaccades. The average population rate at the retinotopic position of the visual target (black curves) and the anti-saccade target position (gray curves) are shown for excitatory neurons in layers $4,2 / 3$ and $5 \mathrm{r}$ and for inhibitory neurons in layer $2 / 3$. Only correct trials were taken into account. Traces of single trials started with the stimulus presentation and ended with the time of the saccade.

Excitatory neurons in layer 4 and layer $2 / 3$ showed a clear selection of the visual target followed by a selection of the saccade target position in antisaccade trials. In some trials, layer 4 selected the visual input a second time, after it had been correctly transformed into an antisaccade quasi-visual signal. This second visual selection was a result of the prospective visual saliency in layer 4, which always "preselected" a new target, as soon as the attentional signal was established at the target previously selected. The activities of layer 4 and layer $2 / 3$ neurons were directly comparable with "type I" neurons reported in experiments [Sato and Schall (2003), their Fig. 2]. Activity in neurons in layer 4 of the model was inhibited before the time of the saccade, whereas layer $2 / 3$ neurons fired until the time of the saccade. A similar inhibition of visual activity was reported in visual prosaccades (Bruce and Goldberg, 1985).

Layer 5r neurons clearly selected the endpoint of the saccade, but had only little activity at the position of the visual target in antisaccade trials. Similar neurons were observed by Sato and Schall (2003, their Fig. 3) and were called "type II."

The FEF model also exhibited neuronal activity that has not been reported in experiments. Inhibitory neurons in layer $2 / 3$ (Fig. $7 B$, bottom) did not show any selectivity for the position of the target in prosaccade trials. However, in antisaccade trials, they signaled the shift of the attention. This specific response was caused by input from the REC module, and it should be observable in experiments.

The mapping through quasi-visual input in layer 4 was well illustrated in experiments involving a delayed memory antisaccade task (Amemori and Sawaguchi, 2006). A visual prosaccade
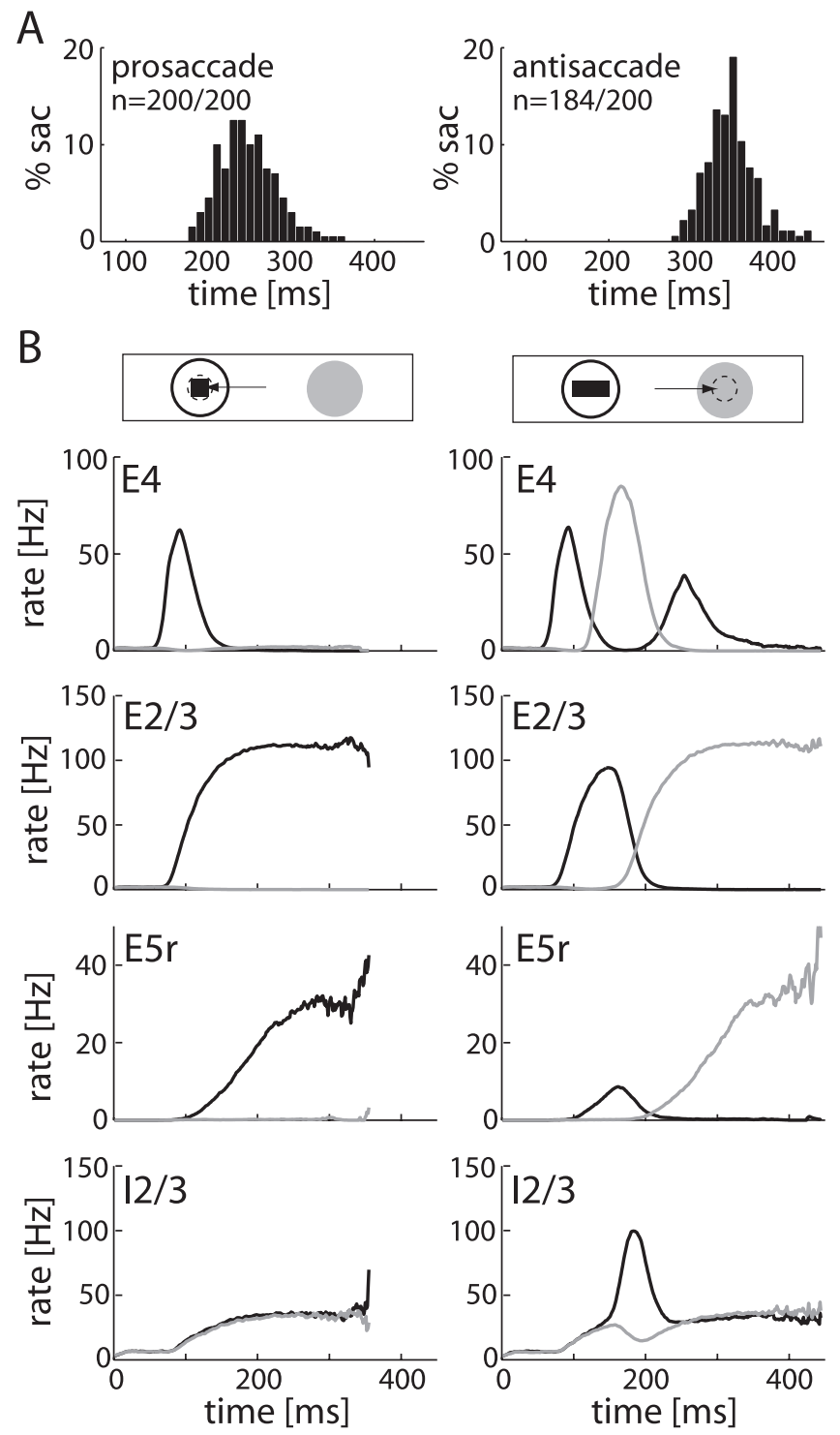

C
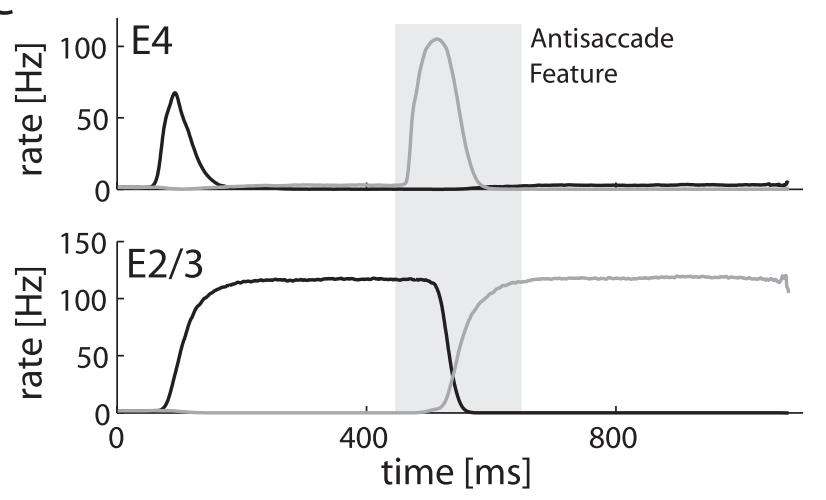

Figure 7. Comparison of prosaccade versus antisaccade trials. $\boldsymbol{A}$, Distributions of reaction times for the two tasks. $\boldsymbol{B}$, Population firing rates at the retinotopic position of the visual target (black curves) and the position mirrored at the vertical meridian (gray curves) are shown for several populations. All traces are averages over all correct trials. Excitatory neurons in layer 4 and $2 / 3$ clearly selected the visual target and the saccade target position in antisaccade trials. Neurons in layer $5 \mathrm{r}$ mainly selected the saccade target and showed only little activity for the visual target in antisaccade trials. Inhibitory neurons in layer $2 / 3$ reflected the recognition signal. They had a differential activity between the two positions only in the antisaccade task. $\boldsymbol{C}$, Activity of neurons in layers 4 and $2 / 3$ in a delayed memory antisaccade task. Same conventions as above. The gray shaded area indicates the time during which the antisaccade feature was presented. Traces are aligned to the onset of the first visual input. 
stimulus was shown at the beginning of the trial. In the middle of the delay period, a second input was shown that signaled the antisaccade rule. Hence, the task was to remap the motor plan according to that input in the middle of the delay period. The network made 99\% (199 of 200) correct eye movements. Figure $7 C$ illustrates the activation of neurons in layers 4 and $2 / 3$ of the model. Note the quasi-visual signal in layer 4 that was induced at the time of the remapping (Amemori and Sawaguchi, 2006, their Fig. 5B). Activity of neurons in layer $2 / 3$ signaled the "intention of a movement" [Zhang and Barash (2000), their Fig. 4; Amemori and Sawaguchi (2006), their Fig. 5A].

In a covert attention task, the model layer $2 / 3$ neurons reproduced well the experimental results of memory neurons [Thompson et al. (2005a), their Fig. 3], whereas visual neurons in layer 4 failed to show the prolonged firing measured in the FEF. The ramping neurons in layer $5 \mathrm{r}$ were suppressed if the monkey had to fixate [Thompson et al. (2005a), their Fig. 4]. Hence, our model is in line with the finding that covert attention can be controlled by the FEF without evoking any motor activity.

\section{Scanning of a constant visual scene}

Finally, we simulated the scanning of a constant visual scene. The network looked at a visual stimulus that contained typically six targets, which differed in their intensity and hence in their input strength to layer 4. Five different configurations of the network were each simulated for a period of $60 \mathrm{~s}$. These networks differed in the random distribution of weights and in their random external inputs. A $5 \mathrm{~s}$ excerpt of a fixation pattern is shown in Figure $8 \mathrm{~A}$.

During the $60 \mathrm{~s}$ trials, the network made on average $202 \pm 4$ saccades. A total of 1011 saccades were recorded for the five different configurations of the network. The resulting distribution of fixation times is shown in Figure 8 B. The average fixation time was $296 \pm 98 \mathrm{~ms}$ with a median of $267 \mathrm{~ms}$, and the 5 th and 95th percentiles were 213 and $509 \mathrm{~ms}$, respectively. Similar distributions were observed in scene viewing (Henderson, 2003) and reading (Rayner, 1998) in humans and also in viewing of natural scenes in monkeys Schiller et al., 2004). Although the shape of the fixation time distribution is very similar [Schiller et al. (2004), their Fig. 2], the average fixation times of our model lie between the slower average times for humans and the faster times recorded in macaque monkeys.

At each saccade, the retinotopic input to the network was updated to match the new direction of gaze. The relative strength of the inputs was reflected in the number of saccades that were made to a particular target. The majority of saccades (57\%) landed on one of the two targets with input strength 1 . The me-
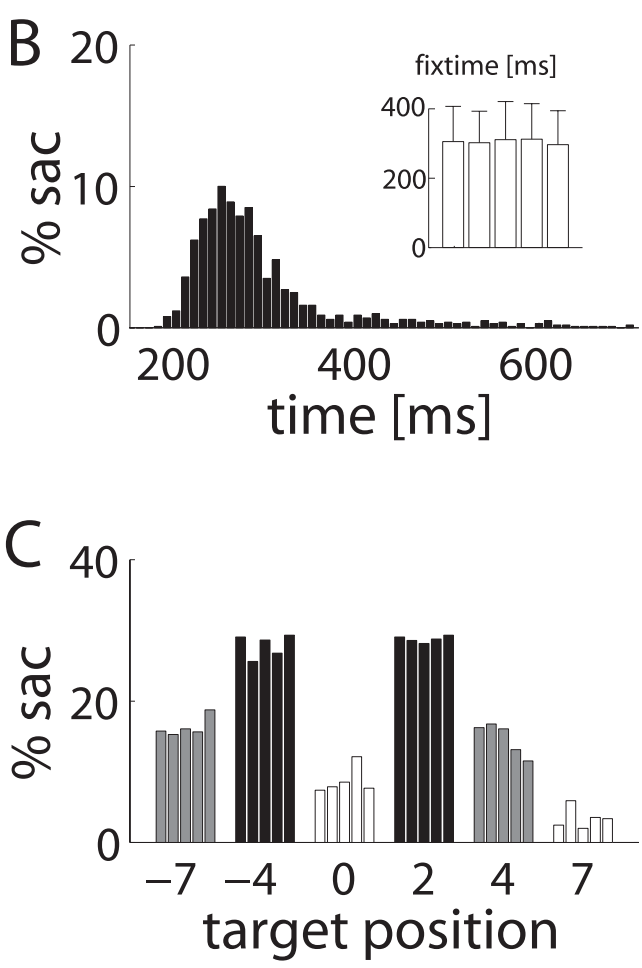

Figure 8. Scanning of a visual scene. $\boldsymbol{A}$, Foveal position as a function of time over a period of $5 \mathrm{~s}$ (gray trace) sampled from $60 \mathrm{~s}$ ocations. $\boldsymbol{B}$, Distribution of fixation times binned in $10 \mathrm{~ms}$ time windows. A total of 1011 saccades entered this distribution (mean \pm SD, $296 \pm 98 \mathrm{~ms}$ ). The inset shows the mean \pm SD for the five simulations of 60 s individually. $C$, Distribution of saccade the of saccades made to the six positions. The probability of selecting each target is shown for each of the five 作

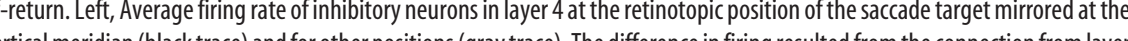
6 s to layer 4 . Middle, Same traces for the simulations, in which the weight of the slow 6 s to layer 4 connection was set to zero. No difference was seen between the different retinotopic positions (same conventions as before). Left, Percentage of return saccades for the five simulations of $60 \mathrm{~s}$ with and without inhibition-of-return (IR).

dium strength targets were fixated in $31 \%$ and the weak targets in $12 \%$ of the trials (Fig. 8 C).

The connection from layer 6 s to the layer 4 inhibitory neurons introduced inhibition-of-return to the behavior of the network and so reduced the probability of gaze being switched back and forth between the two most salient targets. The number of return saccades back to the previously visited target was small (6\%, 60 of 1011). Figure $8 D$ illustrates how the connection from layer $6 \mathrm{~s}$ slightly increased the firing of layer 4 inhibitory neurons at the retinotopic position opposite to the saccade landing position (black trace). When the same task was simulated with the weight,

$$
w_{\mathrm{IR}}^{\mathrm{I} 4 \mathrm{E} 6 \mathrm{~s}}=0
$$

all inhibitory neurons in layer 4 had the same firing pattern and hence there was no inhibition-of-return. The number of return 
saccades then increased to $33 \%$ (Fig. $8 D$, left), whereas the fixation time distribution remained the same (mean \pm SD, $295 \pm$ $100 \mathrm{~ms}$ ). Inhibition-of-return also influenced how frequently targets of different strengths were selected: $62 \%$ of the saccades landed on a target with relative input strength $1,29 \%$ on medium strength, and only $8 \%$ on weak targets. The example of the inhibition-of-return illustrates how the visual selection could be influenced not only with respect to the currently attended position, but also with respect to the last saccade made.

\section{Discussion}

The detailed model of the cortical area FEF presented here incorporated the layered structure of neocortex and used realistic spiking neurons. It showed that a canonical circuit derived from primary visual cortex of the cat could, with relatively few modifications, be used to control eye movements in a variety of tasks seen in primate area FEF.

The model was able to make normal saccades to targets presented briefly in the periphery and was able to scan an array of visual targets. A separate fixation input suppressed saccades and allowed the model to perform a delayed memory saccade task. The mapping of visual targets to the saccade output could be changed according to a given rule. The effect of this rule input was illustrated in a task in which the visual-to-oculomotor transformation depended on the feature of the target. A retinotopic array of feature detectors that performed a feature recognition (the REC module) provided an input to the FEF network. All spatial transformations occurred through remapping of signals in layer 4, which gave rise to so-called quasi-visual activity (Barash, 2003). The model was able to reproduce single cell as well as behavioral data from experiments in awake monkeys. The detailed and biologically realistic functional architecture of the model not only provides plausible mechanisms for existing experimental results but makes precise predictions for future experiments.

\section{A single local circuit for visual selection, attention, and eye movements?}

The compression of function into the single cortical area FEF is, of course, an idealization and simplification of the interactive network of cortical areas and subcortical structures involved in the control of eye movements (Büttner and Büttner-Ennever, 2005). However, all neurons within the model were functionally related to real cortical neurons recorded within the FEF. Visual saliency and target selection (Mohler et al., 1973; Schall et al., 1995b; Schall, 2004; Thompson and Bichot, 2005), short-term memory responses (Bruce and Goldberg, 1985), attention related activity (Thompson et al., 2005a), saccadic activity (Bruce and Goldberg, 1985; Segraves and Park, 1993), and fixation-related responses (Hanes et al., 1998; Hasegawa et al., 2004) were described in FEF experiments and were captured by the model network.

The FEF model included a functional segregation between different layers, as is observed in early visual areas. Nothing is known about the local circuit in FEF, and the lack of direct data on the layered position of FEF neurons in recordings from awake monkeys makes it impossible to answer conclusively the question of the laminar segregation of functions. As in the laminar segregation of receptive field types in primary visual cortex, the true segregation of functional properties may not be as strict as the model implies. For example, cells projecting to the SC, which are presumably located in layer 5 (Leichnetz et al., 1981), were found to show visual and memory activity as well (Sommer and Wurtz,
2000, 2001). Although the strict functional segregation of the model is not in line with these findings, it would not violate the principles of operation of the model to include in layer 5 some relay neurons that show visual and memory activity. The strong prediction of the FEF model, however, is that the local circuit in primate FEF will follow the same principles of cell-type and laminar-specific connections as those determined from the extensive studies of cat primary visual cortex.

Electrical stimulation experiments (Moore and Armstrong, 2003; Moore and Fallah, 2004; Armstrong et al., 2006; Ruff et al., 2006; Armstrong and Moore, 2007) and neuronal responses (Thompson et al., 2005a) have clearly demonstrated the role of the FEF in guiding attention, as exemplified by the role of layer $2 / 3$ in the model. This makes an interesting interpretation with respect to the premotor theory of spatial visual attention (Rizzolatti et al., 1987): the FEF model can control covert attention without motor activity (Thompson et al., 2005a). The connection of layer $2 / 3$ to the REC module, which is presumably located in inferotemporal cortex, is in line with the observation that the projections of FEF to temporal areas such as the temporal-occipital area and the visual cortical area V4 arise mainly from pyramidal neurons in the superficial layers (Schall et al., 1995a; Barone et al., 2000).

\section{Anatomical considerations}

The laminar connectivity of the model was based directly on the canonical circuit of cat visual cortex (Douglas et al., 1989; Binzegger et al., 2004) and tuned to perform the function of the primate FEF. Despite this functional tuning, the anatomical structure of the FEF circuit was well conserved, and the function of the FEF was robust to small changes (up to 10\%) in the connectivity pattern. The connections within the individual layers were stronger than the connections between layers in the feedforward loop (layer 4-layer 2/3-layer 5-layer 6-layer 4). The intralaminar connections within layer 5 , however, were much stronger than in cat visual cortex. The main reason for the large number of connections in layer 5 was the required bursting behavior that was entirely attributable to recurrent connections and not a result of the biophysical properties of single neurons.

Some interlaminar connections were considerably stronger than expected from cat visual cortex. The connections from layer $5 b$ to layer $2 / 3$ excitatory and inhibitory neurons and the connection from layer 6 s to layer 4 inhibitory neurons all required stronger connections than predicted from the cat cortex. Interestingly, all three connections were involved in controlling the network activity after saccades. Remarkably, just these few changes in the strength of connections of neurons in layers 5 and 6 of allowed the local circuit of cat visual cortex to function as a primate FEF area.

\section{Rule input as top-down bias for visual selection}

The connection from layer 6 a to layer 4 provided a dynamic top-down bias for the function of visual selection. It depended on the current focus of attention and the last saccade. In the absence of visual targets, this bias induced a quasi-visual signal. Neuronal activity related to a prosaccade versus antisaccade rule was observed in prefrontal areas (Everling and DeSouza, 2005; Amemori and Sawaguchi, 2006), and a top-down saliency on the visual selection in the FEF was also reported (Thompson et al., 2005b). A typical example of a top-down bias would be the leftto-right bias in humans in Western culture attributable to reading (Spalek and Hammad, 2005). Although the anatomical connection enabling the FEF model to perform antisaccades was 
hardwired, as expected for highly learned tasks, it was dynamically activated through the rule input. Other remappings, such as making saccades to the midpoint between the target and the fixation point, could be implemented in the same way.

Whether the rule input targets layer 6 of the FEF is an open question. The strength of the connection from layer 6 to layer 4 in visual cortex (Binzegger et al., 2004) suggests that this connection could provide a powerful modulation that directly acts on the input layer of the FEF. Responses of visual neurons during voluntary saccades in the dark (Bruce and Goldberg, 1985) and quasi-visual responses in area LIP during antisaccades (Zhang and Barash, 2000) indeed suggest such a mechanism that acts via a quasi-sensory input. Firing-rate models of task-specific sensorimotor mappings can be achieved through learned spatially selective connections in which task modulated sensory neurons project directly onto the motor units (Salinas, 2004a,b).

\section{Other models of the FEF}

Of the three recent FEF models, the internal update of short-term memory during saccades (Mitchell and Zipser, 2003) and the "reentry hypothesis" of attentional influence of FEF on area V4 (Hamker, 2005) focused on computational aspects that were not addressed by our model. Hence, they are not directly comparable. A similar layered structure as in our model was used by Brown et al. (2004). In contrast to our model, layer 4 of their model only normalized the incoming visual input without any additional computation. Layers $2 / 3$ and 5 were similar to our model, but organized in feature-specific zones. However, the feature specificity predicted by their model was reported in the FEF only in one overtrained paradigm (Bichot et al., 1996). In the model of Brown et al. (2004) a rule was implemented by the connection from layer 6 to layer 2/3; however, the layer 6 to layer 4 connection in our model reflects better the known anatomical connections (Binzegger et al., 2004) and is consistent with quasi-visual signals (Barash, 2003).

A ramping-to-threshold behavior that is very similar to ramping neurons in layer 5 was modeled recently in the context of saccade generation (Lo and Wang, 2006). In the study by Lo and Wang (2006), ramping occurred in the cortex, but, unlike in our model, the saccadic burst itself was produced in the SC. In general, layered structures do have computational advantages (Douglas and Martin, 2004; Haeusler and Maass, 2007), but cortex may have additional constraints, such as efficient developmental mechanisms and a requirement for multiple parallel and distributed processing, that also have strong influence on the final form of the circuit .

\section{General conclusions and outlook}

The local circuit model of the FEF presented in this paper is one of the few models of a layered cortical microcircuit that tries to simulate real cortical behavior. The realistic, layered organization of the model and its implementation with spiking neurons allowed us to compare results directly with physiological data and ensured that the computational strategy of the model was biologically feasible. One main advantage of the layered structure was the possibility to have separate, stable functions within single layers as proposed by Douglas and Martin (2004). The detailed structure of the model makes clear predictions on the functional role of the microcircuit of the FEF. Many important assumptions, such as the behavior of inhibitory neurons and the role of layer 6, have yet to be tested by experiments. Thus, the model not only offers plausible, biologically based mechanisms that underlie a rich repertoire of saccadic eye movement behavior, but also makes specific predictions about the structure of the circuits to be found in primate FEF and the functional role of particular neuronal elements of the network.

The dynamic control of the visual selection via an attentionand saccade-dependent rule is a highly flexible mechanism. It could be used in other tasks such as reading in humans that involve the coupling of a top-down saliency and a premotor response. Finally, the model demonstrates that a cortical circuit based on a primary visual area in the cat requires only few changes in its connectivity to be able to compute the very different functions of the primate prefrontal area FEF. The general principle of the canonical cortical circuit is strong recurrent, intralaminar connections and rather weak ones between layers. This principle of function is the basis of a powerful and flexible computational circuit.

\section{References}

Amador N, Schlag-Rey M, Schlag J (1998) Primate antisaccades. I. Behavioral characteristics. J Neurophysiol 80:1775-1786.

Amemori K-I, Sawaguchi T (2006) Rule-dependent shifting of sensorimotor representation in the primate prefrontal cortex. Eur J Neurosci 23:1895-1909.

Armstrong KM, Moore T (2007) Rapid enhancement of visual cortical response discriminability by microstimulation of the frontal eye field. Proc Natl Acad Sci USA 104:9499-9504.

Armstrong KM, Fitzgerald JK, Moore T (2006) Changes in visual receptive fields with microstimulation of frontal cortex. Neuron 50:791-798.

Barash S (2003) Paradoxical activities: insight into the relationship of parietal and prefrontal cortices. Trends Neurosci 26:582-589.

Barone P, Batardiere A, Knoblauch K, Kennedy H (2000) Laminar distribution of neurons in extrastriate areas projecting to visual areas V1 and V4 correlates with the hierarchical rank and indicates the operation of a distance rule. J Neurosci 20:3263-3281.

Bichot NP, Schall JD, Thompson KG (1996) Visual feature selectivity in frontal eye fields induced by experience in mature macaques. Nature 381:697-699.

Binzegger T, Douglas RJ, Martin KAC (2004) A quantitative map of the circuit of cat primary visual cortex. J Neurosci 24:8441-8453.

Bizzi E (1967) Discharge of frontal eye field neurons during eye movements in unanesthetized monkeys. Science 157:1588-1590.

Brown JW, Bullock D, Grossberg S (2004) How laminar frontal cortex and basal ganglia circuits interact to control planned and reactive saccades. Neural Networks 17:471-510.

Bruce CJ, Goldberg ME (1985) Primate frontal eye fields. I. Single neurons discharging before saccades. J Neurophysiol 53:603-635.

Bruce CJ, Goldberg ME, Bushnell MC, Stanton GB (1985) Primate frontal eye fields. II. Physiological and anatomical correlates of electrically evoked eye movements. J Neurophysiol 54:714-734.

Büttner U, Büttner-Ennever JA (2005) Present concepts of oculomotor organization. Prog Brain Res 151:1-42.

Dias EC, Segraves MA (1999) Muscimol-induced inactivation of monkey frontal eye field: effects on visually and memory-guided saccades. J Neurophysiol 81:2191-2214.

Dias EC, Kiesau M, Segraves MA (1995) Acute activation and inactivation of macaque frontal eye field with GABA-related drugs. J Neurophysiol 74:2744-2748.

Dominey PF, Arbib MA (1992) A cortico-subcortical model for generation of spatially accurate sequential saccades. Cereb Cortex 2:153-175.

Douglas RJ, Martin KA (1991) A functional microcircuit for cat visual cortex. J Physiol (Lond) 440:735-769.

Douglas RJ, Martin KA (2004) Neuronal circuits of the neocortex. Annu Rev Neurosci 27:419-451.

Douglas RJ, Martin KAC, Whitteridge D (1989) A canonical microcircuit for neocortex. Neural Comput 1:480-488.

Everling S, DeSouza JFX (2005) Rule-dependent activity for prosaccades and antisaccades in the primate prefrontal cortex. J Cogn Neurosci 17:1483-1496.

Everling S, Dorris MC, Klein RM, Munoz DP (1999) Role of primate superior colliculus in preparation and execution of anti-saccades and prosaccades. J Neurosci 19:2740-2754. 
Ferrier D (1874) Experiments on the brain of monkeys. I. Proc R Soc Lond B Biol Sci 23:409-430.

Goldberg ME, Bruce CJ (1990) Primate frontal eye fields. III. Maintenance of a spatially accurate saccade signal. J Neurophysiol 64:489-508.

Haeusler S, Maass W (2007) A statistical analysis of information-processing properties of lamina-specific cortical microcircuit models. Cereb Cortex $17: 149-162$.

Hamker FH (2005) The reentry hypothesis: the putative interaction of the frontal eye field, ventrolateral prefrontal cortex, and areas V4, IT for attention and eye movement. Cereb Cortex 15:431-447.

Hanes DP, Patterson WF, II, Schall JD (1998) Role of frontal eye fields in countermanding saccades: visual, movement, and fixation activity. J Neurophysiol 79:817-834.

Hasegawa RP, Peterson BW, Goldberg ME (2004) Prefrontal neurons coding suppression of specific saccades. Neuron 43:415-425.

Henderson JM (2003) Human gaze control during real-world scene perception. Trends Cogn Sci 7:498-504.

Huerta MF, Krubitzer LA, Kaas JH (1986) Frontal eye field as defined by intracortical microstimulation in squirrel monkeys, owl monkeys, and macaque monkeys. I. Subcortical connections. J Comp Neurol 253:415-439.

Huerta MF, Krubitzer LA, Kaas JH (1987) Frontal eye field as defined by intracortical microstimulation in squirrel monkeys, owl monkeys, and macaque monkeys. II. Cortical connections. J Comp Neurol 265:332-361.

Kisvarday ZF, Cowey A, Smith AD, Somogyi P (1989) Interlaminar and lateral excitatory amino acid connections in the striate cortex of monkey. J Neurosci 9:667-682.

Kritzer MF, Goldman-Rakic PS (1995) Intrinsic circuit organization of the major layers and sublayers of the dorsolateral prefrontal cortex in the rhesus monkey. J Comp Neurol 359:131-143.

Leichnetz GR, Spencer RF, Hardy SGP, Astruc J (1981) The prefrontal corticotectal projection in the monkey; an anterograde and retrograde horseradish peroxidase study. Neuroscience 6:1023-1041.

Lo C-C, Wang X-J (2006) Cortico-basal ganglia circuit mechanism for a decision threshold in reaction time tasks. Nat Neurosci 9:956-963.

Lynch JC, Tian JR (2005) Cortico-cortical networks and cortico-subcortical loops for the higher control of eye movements. Prog Brain Res 151:461-501.

Mitchell JF, Zipser D (2003) Sequential memory-guided saccades and target selection: a neural model of the frontal eye fields. Vision Res 43:2669-2695.

Mohler CW, Goldberg ME, Wurtz RH (1973) Visual receptive fields of frontal eye field neurons. Brain Res 61:385-389.

Moore T, Armstrong KM (2003) Selective gating of visual signals by microstimulation of frontal cortex. Nature 421:370-373.

Moore T, Fallah M (2004) Microstimulation of the frontal eye field and its effects on covert spatial attention. J Neurophysiol 91:152-162.

Parthasarathy HB, Schall JD, Graybiel AM (1992) Distributed but convergent ordering of corticostriatal projections: analysis of the frontal eye field and the supplementary eye field in the macaque monkey. J Neurosci 12:4468-4488.

Petrides M (2005) Lateral prefrontal cortex: architectonic and functional organization. Philos Trans R Soc B Biol Sci 360:781-795.

Rayner K (1998) Eye movements in reading and information processing: 20 years of research. Psychol Bull 124:371-422.

Riesenhuber M, Poggio T (1999) Hierarchical models of object recognition in cortex. Nat Neurosci 2:1019-1025.

Rizzolatti G, Riggio L, Dascola I, Umilta C (1987) Reorienting attention across the horizontal and vertical meridians: evidence in favor of a premotor theory of attention. Neuropsychologia 25:31-40.

Robinson DA, Fuchs AF (1969) Eye movements evoked by stimulation of frontal eye fields. J Neurophysiol 32:637-648.

Roesch MR, Olson CR (2005) Neuronal activity dependent on anticipated and elapsed delay in macaque prefrontal cortex, frontal and supplementary eye fields, and premotor cortex. J Neurophysiol 94:1469-1497.

Ruff CC, Blankenburg F, Bjoertomt O, Bestmann S, Freeman E, Haynes J-D, Rees G, Josephs O, Deichmann R, Driver J (2006) Concurrent TMSfMRI and psychophysics reveal frontal influences on human retinotopic visual cortex. Curr Biol 16:1479-1488.

Salinas E (2003) Background synaptic activity as a switch between dynamical states in a network. Neural Comput 15:1439-1475.

Salinas E (2004a) Fast remapping of sensory stimuli onto motor actions on the basis of contextual modulation. J Neurosci 24:1113-1118.

Salinas E (2004b) Context-dependent selection of visuomotor maps. BMC Neuroscience 5:47.

Sato TR, Schall JD (2003) Effects of stimulus-response compatibility on neural selection in frontal eye field. Neuron 38:637-648.

Schall JD (2004) On the role of frontal eye field in guiding attention and saccades. Vision Res 44:1453-1467.

Schall J, Morel A, King D, Bullier J (1995a) Topography of visual cortex connections with frontal eye field in macaque: convergence and segregation of processing streams. J Neurosci 15:4464-4487.

Schall JD, Hanes DP, Thompson KG, King DJ (1995b) Saccade target selection in frontal eye field of macaque. I. Visual and premovement activation. J Neurosci 15:6905-6918.

Schiller PH, Slocum WM, Carvey C, Tolias AS (2004) Are express saccades generated under natural viewing conditions? Eur J Neurosci 20:2467-2473.

Schnyder H, Reisine H, Hepp K, Henn V (1985) Frontal eye field projection to the paramedian pontine reticular formation traced with wheat germ agglutinin in the monkey. Brain Res 329:151-160.

Segraves MA (1992) Activity of monkey frontal eye field neurons projecting to oculomotor regions of the pons. J Neurophysiol 68:1967-1985.

Segraves MA, Park K (1993) The relationship of monkey frontal eye field activity to saccade dynamics. J Neurophysiol 69:1880-1889.

Sommer MA, Wurtz RH (2000) Composition and topographic organization of signals sent from the frontal eye field to the superior colliculus. J Neurophysiol 83:1979-2001.

Sommer MA, Wurtz RH (2001) Frontal eye field sends delay activity related to movement, memory, and vision to the superior colliculus. J Neurophysiol 85:1673-1685.

Spalek TM, Hammad S (2005) The left-to-right bias in inhibition of return is due to the direction of reading. Psychol Sci 16:15-18.

Stanton GB, Deng SY, Goldberg ME, McMullen NT (1989) Cytoarchitectural characteristic of the frontal eye fields in macaque monkeys. J Comp Neurol 282:415-427.

Thompson KG, Bichot NP (2005) A visual salience map in the primate frontal eye field. Prog Brain Res 147:251-262.

Thompson KG, Biscoe KL, Sato TR (2005a) Neuronal basis of covert spatial attention in the frontal eye field. J Neurosci 25:9479-9487.

Thompson KG, Bichot NP, Sato TR (2005b) Frontal eye field activity before visual search errors reveals the integration of bottom-up and top-down salience. J Neurophysiol 93:337-351.

Umeno MM, Goldberg ME (2001) Spatial processing in the monkey frontal eye field. II. Memory responses. J Neurophysiol 86:2344-2352.

Zhang M, Barash S (2000) Neuronal switching of sensorimotor transformations for antisaccades. Nature 408:971-975. Vol. 27, No. 34 